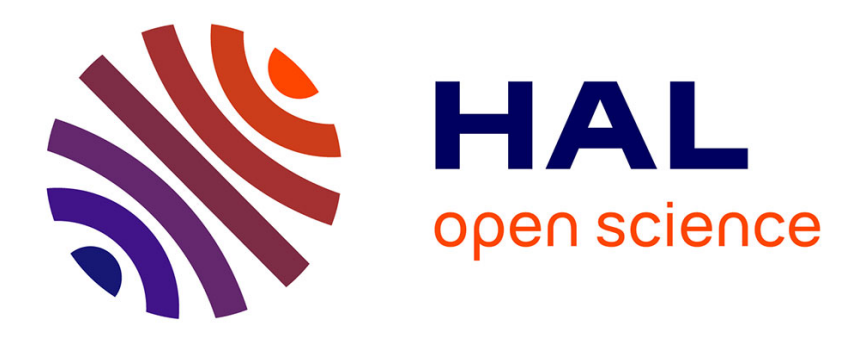

\title{
Extending morphological signatures for visual pattern recognition
}

Sébastien Lefèvre

\section{To cite this version:}

Sébastien Lefèvre. Extending morphological signatures for visual pattern recognition. International Workshop on Pattern Recognition in Information Systems (PRIS), 2007, Funchal, Portugal. pp.79-88. hal-00516078

\section{HAL Id: hal-00516078 https://hal.science/hal-00516078}

Submitted on 13 Nov 2019

HAL is a multi-disciplinary open access archive for the deposit and dissemination of scientific research documents, whether they are published or not. The documents may come from teaching and research institutions in France or abroad, or from public or private research centers.
L'archive ouverte pluridisciplinaire HAL, est destinée au dépôt et à la diffusion de documents scientifiques de niveau recherche, publiés ou non, émanant des établissements d'enseignement et de recherche français ou étrangers, des laboratoires publics ou privés. 


\title{
Extending Morphological Signatures for Visual Pattern Recognition
}

\author{
Sébastien Lefèvre \\ LSIIT, CNRS / University Louis Pasteur - Strasbourg I \\ Parc d'Innovation, Bvd Brant, BP 10413 \\ 67412 Illkirch Cedex, France \\ lefevredsiit.u-strasbg.fr
}

\begin{abstract}
Morphological signatures are powerful descriptions of the image content which are based on the framework of mathematical morphology. These signatures can be computed on a global or local scale: they are called pattern spectra (or granulometries and antigranulometries) when measured on the complete images and morphological profiles when related to single pixels. Their goal is to measure shape distribution instead of intensity distribution, thus they can be considered as a relevant alternative to classical intensity histograms, in the context of visual pattern recognition.

A morphological signature (either a pattern spectrum or a morphological profile) is defined as a series of morphological operations (namely openings and closings) considering a predefined pattern called structuring element. Even if it can be used directly to solve various pattern recognition problems related to image data, the simple definitions given in the binary and grayscale cases limit its usefulness in many applications.

In this paper, we introduce several 2-D extensions to the classical 1-D morphological signature. More precisely, we elaborate morphological signatures which try to gather more image information and do not only include a dimension related to the object size, but also consider on a second dimension a complementary information relative to size, intensity or spectral information. Each of the 2-D morphological signature proposed in this paper can be defined either on a global or local scale and for a particular kind of images among the most commonly ones (binary, grayscale or multispectral images). We also illustrate these signatures by several real-life applications related to object recognition and remote sensing.

Keywords: Mathematical Morphology, Granulometry, Pattern spectrum, Morphological profile, Visual Pattern Recognition
\end{abstract}

\section{Introduction}

When applied on visual information such as digital images, pattern recognition and data mining techniques are very dependent on the accuracy of the image features they rely on. The image analysis and processing community has proposed from several decades a large variety of features to represent precisely the content of an image. The features which have received the most attention from the image scientists and engineers are context-independent, i.e. they can be used for a various panel of problems and a wide range of images. 
Among these features, the histogram is certainly the most plebiscited and represents the probability density function of the intensity values in the image. It can be analysed through various measures such as moments, entropy, uniformity, or other information criteria. However it does not take into account the spatial relationships between pixels.

On the contrary, approaches such as pattern spectra, granulometries, or morphological profiles are built from series of morphological filtering operations and thus involve a spatial information. They can be applied either on a local scale or a global scale. In the first case, the differential morphological profiles introduced in [1] have shown their interest to deal with classification of remote sensing data. On a global scale, pattern spectra and granulometries are widely known in the image analysis community [2]. However all these morphological measures are limited to a single evolution curve and so cannot consider simultaneously several dimensions. Some attempts have been made to generate vectorial granulometries [3] or vectorial covariances [4], but they are more vectorial extensions than multidimensional extensions.

In this paper, we propose several extensions to build 2-D series of morphological measures. We first give the necessary definitions and show the similarity between the different morphological measures which have been proposed in the literature. We then introduce three different 2-D signatures, related to size-size, size-intensity, and sizespectrum information. The interest of our contribution is then illustrated by several applications related to object recognition and remote sensing. We believe however that these extensions can be used as appropriate image features to solve a larger panel of pattern recognition and image mining problems.

\section{Preliminary definitions}

Morphological signatures are tools provided by the framework of Mathematical Morphology (MM) and known as pattern spectra (and granulometries / antigranulometries) or morphological profiles when computed on a global or a local scale respectively. The theory of MM has been introduced by Matheron and Serra [5] in the mid sixties and has been extensively used for 40 years particulary for spatial-based image analysis. The theoretical framework of complete lattices [6] is commonly used to elaborate morphological operators on monovalued (binary or grayscale) images. Multivalued (colour or multispectral) images require the choice of a specific vectorial ordering [7]. In this section, we will recall the definitions of the basic morphological operators from which we can then give the formulation of the morphological signature.

\subsection{Fundamental operators}

Let $f: E \rightarrow T$ represents the image to be morphologically processed, with $E$ being the discrete coordinate grid (usually $\mathbb{N}^{2}$ for a 2-D image) while $T$ represents the set of possible image values. In the case of a binary image, $T=\{0,1\}$ where the objects and the background are respectively represented by values equal to 1 and 0 . In the case of a grayscale image, $T$ is generally a subset of $\mathbb{Z}$, for instance $[0,255]$. Finally, $\mathbb{Z}^{n}$ is considered for $T$ in case of multispectral images. Most of the available operators within the MM framework are applied on an input image $f$ (either binary, grayscale 
or multispectral) and rely also on a predefined matching pattern $B$, called structuring element (SE). In this paper we assume that only flat structuring elements are involved, thus resulting in the definition of $B$ as a subset of $E$.

Based on these notations, the definitions of the two basic morphological operators (namely dilation and erosion) are given by:

$$
\begin{aligned}
& \delta_{B}(f)(x, y)=\bigvee_{(r, s) \in B} f(x-r, y-s), \quad(x, y) \in E \\
& \epsilon_{B}(f)(x, y)=\bigwedge_{(r, s) \in B} f(x+r, y+s), \quad(x, y) \in E
\end{aligned}
$$

In other words, the dilation $\delta_{B}(f)$ results in an image where each pixel $(x, y)$ is associated to the local maximum of $f$ in the neighbourhood defined by the SE $B$. The erosion is the dual operator, considering the local minimum instead of the local maximum.

We can then derive the opening and closing operators:

$$
\begin{gathered}
\gamma_{B}(f)=\delta_{B}\left(\epsilon_{B}(f)\right) \\
\phi_{B}(f)=\epsilon_{B}\left(\delta_{B}(f)\right)
\end{gathered}
$$

where the two fundamental operators are applied successively to filter the input image: erosion followed by dilation for the opening, dilation followed by erosion for the closing operator, thus resulting in a removal of either local maxima or minima. From these definitions we can observe that the strength of an opening or closing filter depends directly on the size of the SE $B$ : considering an increasing size $\lambda$ for the SE, the openings $\gamma_{\lambda}$ and closings $\phi_{\lambda}$ will remove more and more details from the input image. Indeed the opening and closing operators ensure respectively the extensivity and anti-extensivity properties, in other words $\gamma(f) \subset f$ and $f \subset \phi(f)$. For the sake of conciseness, we will use the operator $\psi$ to represent either $\gamma$ or $\phi$, i.e. the opening or closing operation.

\subsection{Morphological signatures}

As indicated earlier in this paper, the morphological signature can be computed either on a local (i.e. pixel-related) or a global (i.e. image-related) scale, thus resulting in operators respectively known as morphological profile and pattern spectrum (or granulometry / antigranulometry). In order to give the formulation of these different operators, let us write $\Pi_{\psi}(f)$ the series obtained from successive openings or closings:

$$
\Pi_{\psi}(f)=\left\{\Pi_{\psi_{\lambda}}(f) \mid \Pi_{\psi_{\lambda}}(f)=\psi_{\lambda}(f), \forall \lambda \in\{0, \ldots, n\}\right\}
$$

where $\psi_{0}(f)=f$ and $\lambda$ denotes the size of the SE $B$. We assume either $\psi=\gamma$ or $\psi=\phi$. More meaningful series $\Delta_{\psi}(f)$ can be generated with differential measures:

$$
\Delta_{\psi}(f)=\left\{\Delta_{\psi_{\lambda}}(f) \mid \Delta_{\psi_{\lambda}}(f)=\Pi_{\psi_{\lambda}}(f)-\Pi_{\psi_{\lambda-1}}(f), \forall \lambda \in\{1, \ldots, n\}\right\}
$$


Finally we can group together opening and closing series to generate a single $\Pi$ or $\Delta$ series of length $2 n$ :

$$
\begin{aligned}
& \Pi(f)=\left\{\Pi_{c}(f) \mid \Pi_{c}(f)=\left\{\begin{array}{l}
\Pi_{\phi_{-c}}(f), \forall c \in\{-n, \ldots,-1\} \\
\Pi_{\gamma_{c}}(f), \forall c \in\{1, \ldots, n\}
\end{array}\right\}\right. \\
& \Delta(f)=\left\{\Delta_{c}(f) \mid \Delta_{c}(f)=\left\{\begin{array}{l}
\Delta_{\phi_{-c}}(f), \forall c \in\{-n, \ldots,-1\} \\
\Delta_{\gamma_{c}}(f), \forall c \in\{1, \ldots, n\}
\end{array}\right\}\right.
\end{aligned}
$$

The morphological signatures widely used in image analysis and visual pattern recognition can be defined from these different image series, either on a local or a global scale. In the former case, the morphological profile and the well-known differential morphological profile (DMP) are respectively defined as $\Pi(f)(x, y)$ and $\Delta(f)(x, y)$ for a given pixel $(x, y)$ in $f$. In the latter case, the pattern spectrum is built from an analysis of the image series $\Pi(f)$ gathering most frequently the image pixel values through the volume or sum operation, i.e. $\sum_{(x, y) \in E} \Pi(f)(x, y)$. In the particular case of binary images, the image volume can either be computed as the sum of pixel values or as the amount of white pixels (or 1-pixels). Granulometries and antigranulometries are built similarly but are limited respectively to openings and closings, i.e. $\sum_{(x, y) \in E} \Pi_{\gamma}(f)(x, y)$ and $\sum_{(x, y) \in E} \Pi_{\phi}(f)(x, y)$. As for local signatures, the original series $\Pi$ is often replaced in practice by its differential version $\Delta$. Moreover, in order to obtain a shape probability distribution function, a normalisation may be involved, each term being then divided by the initial volume $\sum_{(x, y) \in E} f(x, y)$.

We will now present several 2-D extensions to the principle of morphological signature, using the notations introduced in this section.

\section{2-D morphological signatures}

We have seen previously that the well-known (differential) morphological profile and pattern spectrum (or granulometry / antigranulometry) operators may be written as 1D morphological signatures computed on a local or global scale. Here we will present several 2-D extensions to these signatures, in order to gather more information related either to size, intensity, or spectral information.

\subsection{Size-size signature}

In the $\Pi$ and $\Delta$ series, a unique parameter $\lambda$ is considered for measuring the size evolution. In other words, the resulting series are generated by applying openings and closings with SE $B_{\lambda}$ of increasing size $\lambda$. The $\mathrm{SE} B_{\lambda}$ for a given size $\lambda$ can be obtained by dilating $\lambda$ times the original SE $B$ using a given SE $S$ :

$$
B_{\lambda}=\left\{B_{i} \mid B_{i}=\delta_{S}\left(B_{i-1}\right), \forall i \in\{2, \ldots, \lambda\}\right\}_{\lambda}
$$

with $B_{1}=B$. The SE $S$ can be chosen either as a simple SE such as a square, a cross, or a disc of small size $(3 \times 3$ pixels for instance $)$, or as $B$ itself. 
This definition assuming a single size parameter $\lambda$ prevents us from performing accurate measurement. Indeed, it is not adequate to elliptical or rectangular shapes for instance, where the two independent axes should be taken into account.

So we introduce the 2-D size-size signature which is built using SE $B_{\alpha, \beta}$ with two different size parameters $\alpha$ and $\beta$ that vary independently. The SE $B_{\alpha, \beta}$ for a given pair $(\alpha, \beta)$ of size parameters is obtained by dilating $\alpha$ and $\beta$ times the original SE $B$ with two SE $S$ and $T$ :

$$
B_{\alpha, \beta}=\left\{B_{i, j} \mid B_{i, j}=\delta_{S}\left(B_{i-1, j}\right), B_{i, j}=\delta_{T}\left(B_{i, j-1}\right), \forall i \forall j \in\{2, \ldots, \lambda\}\right\}_{\alpha, \beta}
$$

with $B_{1,1}=B$. A relevant choice for the two SE $S$ and $T$ consists in 1-D SE such as horizontal and vertical lines respectively, with a length of 3 pixels for instance.

It is then possible to generate the $\Pi$ series from this 2-D set of SE $B_{\alpha, \beta}$ :

$$
\Pi_{\psi}(f)=\left\{\Pi_{\psi_{\alpha, \beta}}(f) \mid \Pi_{\psi_{\alpha, \beta}}(f)=\psi_{\alpha, \beta}(f), \forall \alpha \forall \beta \in\{0, \ldots, n\}\right\}
$$

where the application of $\psi$ on $f$ with a SE $B_{\alpha, \beta}$ is noted $\psi_{\alpha, \beta}(f)$ and with the convention $\psi_{0,0}(f)=f$. The $\Delta$ series measures the differential in both size dimensions:

$$
\Delta_{\psi}(f)=\left\{\begin{array}{l}
\Delta_{\psi_{\alpha, \beta}}(f) \mid \Delta_{\psi_{\alpha, \beta}}(f)= \\
2 \Pi_{\psi_{\alpha, \beta}}(f)-\Pi_{\psi_{\alpha-1, \beta}}(f)-\Pi_{\psi_{\alpha, \beta-1}}(f), \forall \alpha \forall \beta \in\{1, \ldots, n\}
\end{array}\right\}
$$

\subsection{Size-intensity signature}

As we can notice in the definitions given in section 2 , the pixel intensity values in grayscale images are used either directly (at local scale) or through the sum operator (at global scale). The original morphological signature does not take into account the distribution of intensity values in the image. This can be seen as a strong limitation as intensity distribution (usually measured by histogram) is a key feature when dealing with pattern recognition in image data.

So we propose to build at the global scale a 2-D morphological signature which takes into account both the size and the intensity distributions. To do so, let us use the Kronecker delta function:

$$
\delta_{i j}= \begin{cases}1 & \text { if } i=j \\ 0 & \text { if } i \neq j\end{cases}
$$

and the histogram function $h_{f}: T \rightarrow \mathbb{Z}$ :

$$
h_{f}(\theta)=\sum_{(x, y) \in E} \delta_{\theta f(x, y)}
$$

Alternatively, we can also use the normalised histogram function $h_{f}^{\prime}: T \rightarrow[0,1]$ where $h_{f}^{\prime}(\theta)=h_{f}(\theta) / \sum_{\theta^{\prime} \in T} h_{f}\left(\theta^{\prime}\right)$. The formulation of the 2-D size-intensity signature is then given by the following $\Pi$ series:

$$
\Pi_{\psi}(f)=\left\{\Pi_{\psi_{\lambda, \theta}}(f) \mid \Pi_{\psi_{\lambda, \theta}}(f)=h_{\psi_{\lambda}(f)}(\theta), \forall \theta \in T, \forall \lambda \in\{0, \ldots, n\}\right\}
$$


and its derivative counterpart is given by the following $\Delta$ series:

$$
\Delta_{\psi}(f)=\left\{\begin{array}{l}
\Delta_{\psi_{\lambda, \theta}}(f) \mid \Delta_{\psi_{\lambda, \theta}}(f)= \\
h_{\psi_{\lambda}(f)-\psi_{\lambda-1}(f)}(\theta), \forall \theta \in T, \forall \lambda \in\{1, \ldots, n\}
\end{array}\right\}
$$

This 2-D size-intensity morphological signature gathers the properties of both the classical morphological signature and the intensity histogram by considering size and intensity information together.

\subsection{Size-spectrum signature}

We have considered until now that the input image was monovalued. In case of multispectral images where $T$ is equal to $\mathbb{Z}^{k}$ or $\mathbb{N}^{k}$, the spectral signature of the image pixels can be involved into the morphological signature. To do so, it is possible to first compute a morphological signature for each of the $k$ spectral components (or bands) and then to combine these $k$ signatures into a single one, either 1-D or 2-D.

In the case of a 2-D signature, the morphological series $\Pi$ can be expressed as:

$$
\Pi_{\psi}(f)=\left\{\Pi_{\psi_{\lambda, \omega}}(f) \mid \Pi_{\psi_{\lambda, \omega}}(f)=\psi_{\lambda}\left(f_{\omega}\right), \forall \omega \in\{1, \ldots, k\}, \forall \lambda \in\{0, \ldots, n\}\right\}
$$

where $f_{\omega}$ is a grayscale image representing the $\omega^{\text {th }}$ spectral component of $f$. In this definition the marginal strategy is used, thus the correlation among the different spectral channels is completely ignored.

To avoid this limitation, it is possible to rather consider a vectorial ordering when applying the morphological operators $\gamma$ and $\phi$ on the multispectral input image $f$. The purpose of a vectorial ordering is to give a way to order vectors and thus to compute vectorial extrema by means of the two operators $\sup ^{v}$ and inf ${ }^{v}$. Assuming a given vectorial ordering, the fundamental dilation and erosion operators are written:

$$
\begin{aligned}
& \delta_{B}^{v}(f)(x, y)=\sup _{(r, s) \in B}^{v} f(x-r, y-s), \quad(x, y) \in E \\
& \epsilon_{B}^{v}(f)(x, y)=\inf _{(r, s) \in B}^{v} f(x+r, y+s), \quad(x, y) \in E
\end{aligned}
$$

and from these operators it is possible to write the vectorial versions of the morphological opening $\gamma^{v}$ and closing $\phi^{v}$ :

$$
\begin{aligned}
\gamma_{B}^{v}(f) & =\delta_{B}^{v}\left(\epsilon_{B}^{v}(f)\right) \\
\phi_{B}^{v}(f) & =\epsilon_{B}^{v}\left(\delta_{B}^{v}(f)\right)
\end{aligned}
$$

The morphological size-spectrum signature can finally be expressed as:

$$
\Pi_{\psi}(f)=\left\{\Pi_{\psi_{\lambda, \omega}}(f) \mid \Pi_{\psi_{\lambda, \omega}}(f)=\left(\psi_{\lambda}^{v}(f)\right)_{\omega}, \forall \omega \in\{1, \ldots, k\}, \forall \lambda \in\{0, \ldots, n\}\right\}
$$

where $\left(\psi_{\lambda}^{v}(f)\right)_{\omega}=\psi_{\lambda}\left(f_{\omega}\right)$ in the specific case of a marginal ordering. For a comprehensive review of vectorial orderings and multivariate mathematical morphology, the reader can refer to [7]. 
Finally, we can also define the differential series $\Delta$ as:

$$
\Delta_{\psi}(f)=\left\{\begin{array}{l}
\Delta_{\psi_{\lambda, \omega}}(f) \mid \Delta_{\psi_{\lambda, \omega}}(f)= \\
\Pi_{\psi_{\lambda, \omega}}(f)-\Pi_{\psi_{\lambda-1, \omega}}(f), \forall \omega \in\{1, \ldots, k\}, \forall \lambda \in\{1, \ldots, n\}
\end{array}\right\}
$$

We have presented here several 2-D extensions to the classical morphological signature. We will now show their potential interest when computed either on a local or a global scale through their use in various applications.

\section{Applications}

We have presented in the previous section several 2-D extensions to the morphological signature, which can be computed either on a local or a global scale. The potential interest of these extensions will be illustrated by a panel of applications made as various as possible and related to building detection / object recognition / pixel classification.

\subsection{Building detection}

The 2-D size-size signature has been used as a global scale to determine optimal attributes for image filtering in the context of building detection in panchromatic remote sensed images. Binary images are obtained by thresholding the initial graylevel images. The filter is a morphological opening whose goal is to remove all details smaller than the estimated size for buildings visible in the binary image.

Figure 1 illustrates the relevance of the proposed 2-D granulometry (using only the 2-D version of the $\Delta_{\gamma}$ series) to automatically determine the optimal SE to be used in the morphological filtering process among sizes $(\alpha, \beta)$ between $(10,10)$ and $(50,50)$. As we can observe, the proposed SE size (here $19 \times 21$ pixels, computed as the highest peak) helps the building detection process by greatly reducing the noisy areas which do not correspond to buildings. The overall process is given in [8].

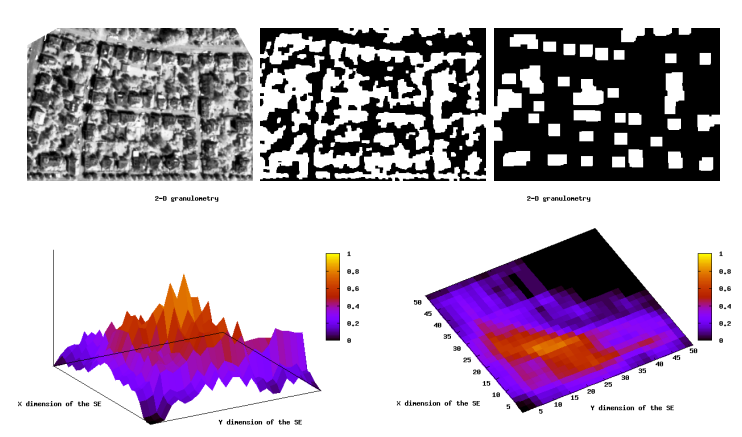

Fig. 1. Relevance of the 2-D size-size global signature to determine optimal parameters for building detection (from left to right and top to bottom): input, unfiltered and filtered images, and curve visualisation (the highest peak determines the optimal parameter). 


\subsection{Object recognition}

In the second application we focus on object recognition in grayscale images. The feature used is the size-intensity signature which has proven to be more discrimant than histogram. The experiments were made on the COIL-20 dataset from Columbia University, which contains 1440 images representing 20 objects under 72 viewpoints.

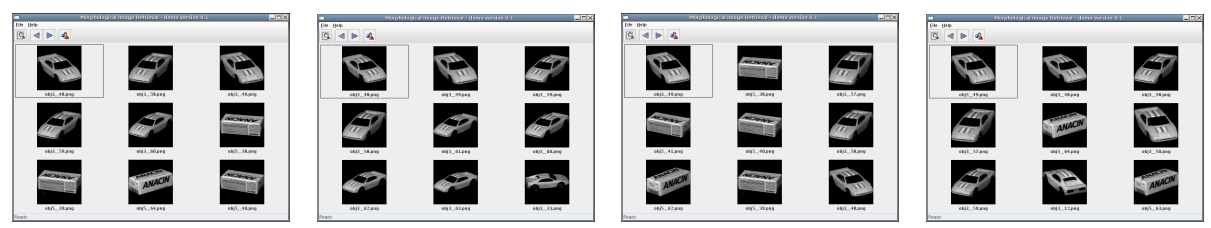

(query \#1, histogram) (query \#1, proposed) (query \#2, histogram) (query \#2, proposed)

Fig. 2. Comparison between the 2-D size-intensity global signature and intensity histogram for object recognition (the input is the top left image).

Considering only intensity distributions through histograms may result in some mistakes between objects, as illustrated by figure 2 . On the contrary, when using morphological size-intensity signatures of same length, the results are better. This signature also leads to more symetrical results, thus the two requests \#1 and \#2 made from a single viewpoint variation of the same object return as first the closest images in the database (obj3_49.png and obj3_48.png).

Some example objects and their respective 2-D size-intensity signatures are given in the figure 3. In this figure, we have considered a combined $\Pi$ series of openings and closings with 256 values for $\theta$ and 25 values for $\lambda$. We can observe the clear difference between the signatures of these three objects.

\subsection{Pixel classification}

The third application illustrates the potential interest of the size-spectrum signature computed on a local scale to deal with multispectral image interpretation in remote sensing through pixel classification. The image used in this experiment is composed of $1100 \times 900$ pixels and 3 spectral components (green, red and near infra-red) and represents an urban area of Strasbourg, France (fig. 4, left). For each pixel $(x, y)$ we consider here a 2-D morphological signature $\Delta$ of size $10 \times 3$ with $\lambda=5$ and $\omega=3$, i.e. 5 openings and 5 closings for 3 spectral bands. A bayesian classification was performed with 5 classes (buildings, roads, water, vegetation, and shadows). The learning set represents $1 \%$ of the complete data and 10 -fold cross-validation was involved. The overall accuracy was about $80-85 \%$ with some classes (buildings, $75 \%$ ) been worse detected than others (water, vegetation, shadows, $95 \%$ ).

In figure 4 is given the original multispectral image in false colours and also an example of classification result using this 2-D size-spectrum local signature. More exhaustive results can be found in [7]. 


\section{Conclusion}

In this paper we have presented several 2-D extensions to the widely used 1-D morphological signature. This signature can be computed on a local or global scale, and is respectively known as (differential) morphological profile and pattern spectrum (or granulometry / antigranulometry). We propose to include another dimension related to size, intensity or spectral information in complement to the initial size distribution, thus obtaining 2-D size-size, size-intensity, and size-spectrum morphological signatures. We finally illustrate the potential interest of these extensions through their use in various applications: building detection, object recognition, and pixel classification.

Future work will include optimisation of the algorithms used to generate the 2-D signatures in order to reduce the computational cost. Another perspective is to exploit in a better way the 2-D signatures using matrix computation. We also consider to elaborate some $n$-D signatures to gather more information in a single signature. Finally, we will have to compare the proposed 2-D morphological signatures with well-known nonmorphological measures, such as wavelets or other textures descriptors.

\section{Acknowledgements}

The author wish to thank his colleagues Erchan Aptoula and Jonathan Weber for some of the experimental works made on remote sensing applications.

\section{References}

1. Benediktsson, J., Pesaresi, M., Arnason, K.: Classification of hyperspectral data from urban areas based on extended morphological profiles. IEEE Transactions on Geoscience and Remote Sensing 43 (2005) 480-491

2. Soille, P.: Morphological Image Analysis : Principles and Applications. Springer-Verlag, Berlin (2003)

3. Wilkinson, M.H.F.: Generalized pattern spectra sensitive to spatial information. In: IAPR International Conference on Pattern Recognition. Volume 1., Quebec City, Canada (2002) 21-24

4. Aptoula, E., Lefèvre, S.: Spatial morphological covariance applied to texture classification. In: International Workshop on Multimedia Content Representation, Classification and Security (IWMRCS), Istanbul, Turkey (2006) 522-529

5. Serra, J.: Image Analysis and Mathematical Morphology. Academic Press (1982)

6. Ronse, C.: Why mathematical morphology needs complete lattices. Signal Processing 21 (1990) 129-154

7. Aptoula, E., Lefèvre, S.: A comparative study on multivariate mathematical morphology. Pattern Recognition (2007) To appear.

8. Lefèvre, S., Weber, J., Sheeren, D.: Automatic building extraction in vhr images using advanced morphological operators. In: IEEE/ISPRS Joint Workshop on Remote Sensing and Data Fusion over Urban Areas, Paris, France (2007) 

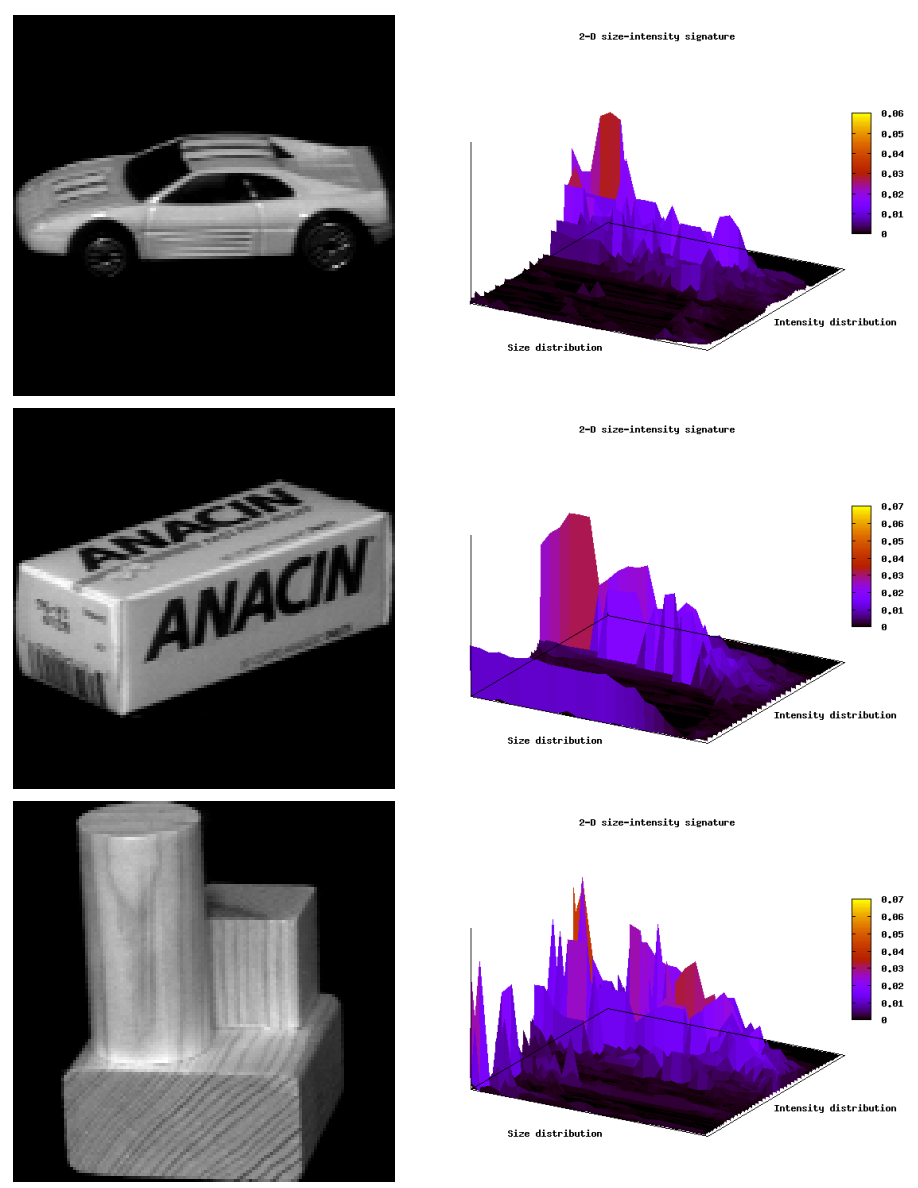

Fig. 3. Relevance of the 2-D size-intensity global signature to discriminate objects in graylevel images: three different objects (left) with relatively similar histograms and their respective 2-D signatures (right).
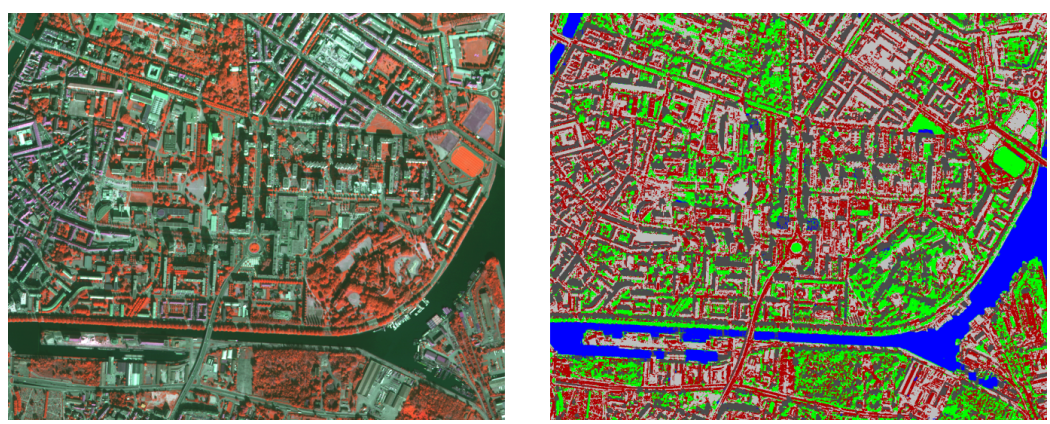

Fig. 4. Relevance of the 2-D size-spectrum local signature to classify pixels in multispectral remote sensed images: input (left) and classified (right) images. 